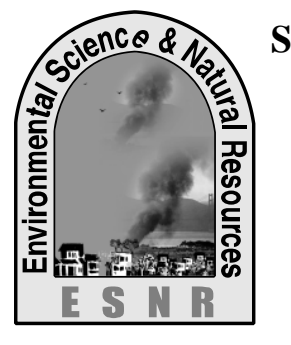

\title{
Screening of Rice Genotypes Based on Root Growth for Salt Tolerance at Germination Stage
}

\author{
D. E. Jharna ${ }^{1^{*}}$, M. A. Hossain ${ }^{2}$, B. L. D. Chowdhury ${ }^{2}$, M. A. A. Lita ${ }^{3}$ and M. M. Islam ${ }^{4}$ \\ ${ }^{1}$ Department of Biochemistry and Food Analysis, Patuakhali Science and Technology University, \\ Patuakhali, \\ ${ }^{2}$ Department of Biochemistry and Molecular Biology, ${ }^{3}$ Department of Agronomy, Bangladesh \\ Agricultural University, Mymensingh, \\ ${ }^{4}$ Biotechnology Division, Bangladesh Institute of Nuclear Agriculture, Mymensingh \\ *Corresponding author: Jharnade@yahoo.com
}

\begin{abstract}
Salinity induced changes in root length and weight under salt tolerant condition. To examine the effect of different degrees of salinity on the root growth of rice, a total of 127 rice genotypes including mostly traditional, cultivated in the coastal regions of Bangladesh and some improved genotypes were used in the study. The study was conducted in petri dish providing $0,6,9,12$ and $15 \mathrm{dS} \mathrm{m}^{-1}$ (deci Siemens) salinity. Based on the performances, genotypes were scored and grouped as highly tolerant (score 1), tolerant (score 3), moderately tolerant (score 5), susceptible (score 7) and highly susceptible (score 9). Salinity stress reduced the root length and weight of rice genotypes. But the extent of root length and weight, varied with genotypes and levels of salinity. Generally in control condition root length and weight was found higher and it gradually decreased with increasing salt concentration. Based on decrease of root dry weight at $15 \mathrm{dS} \mathrm{m}^{-1}$ salinity, 8 genotypes scored 1, 28 scored 3, 40 scored 5, 35 scored 7, and 16 scored 9. Regarding root length, 14 genotypes were found highly tolerant, 18 were tolerant, 27 moderately tolerant, 38 susceptible and 30 were highly susceptible. Based on the score, the relationship between salinity level and tolerances of the different rice genotypes can be understood.
\end{abstract}

Key words: Genotypes, Germination, Rice (Oryza sativa), Root growth and Salt tolerance

\section{Introduction}

Salt stress is a major abiotic stress that leads to a series of changes including plant morphological, physiological, biochemical and molecular changes. Salinity limits the plant growth and profitable crop production worldwide (Kumar et al., 2010; Tavakkoli, 2011). FAO studies reported that $7 \%$ of the arable land affected by salinity (Goldani and Latifi, 2006). It was found that 0.83 Mha of land at coastal Bangladesh were affected by soil salinity at different degrees (CCC, 2007) which reaches up to 1.06 Mha in 2009 (SRDI, 2010). Rice (Oryza sativa L.) is the most important cereal food crop in the world (Ma et al., 2007; Melissa et al., 2009). About one billion people of rural areas in Bangladesh, India and Southeast Asia are depends on rice cultivation. High salt concentration in soil is the major constraint to rice production in Bangladesh and India (Mohammadi-Nejad et al., 2010). It is estimated that a net reduction of 0.5 million metric tons of rice production would take place due to a $0.3 \mathrm{~m}$ sea level rise in coastal areas of Bangladesh (World Bank, 2000).

Salt stress affects many growth parameters i.e., germination percentage, root and shoot length, root and shoot dry matter, root /shoot ratio etc. The detrimental effect of salinity occurs because of osmotic stress and specific ion toxicity (Shrivastava and Kumar, 2015; Zhang et al., 2010; Sosa et al., 2005). Rice is rated as an especially salt-sensitive crop (Mahmud et al., 2017; Gholizadeh and Navabpour, 2011; Zeng and Shannon, 2000). But the response of rice to salinity varies with growth stage. In the most commonly cultivated rice cultivars, young seedlings are very sensitive to salinity (Rad et al., 2012; Ferdose et al., 2009; Shereen et al., 2005; Flowers and Yeo, 1981). According to the classification of crop tolerance to salinity, the rice crop is within the sensitive division from 0 to $8 \mathrm{dS} \mathrm{m}^{-1}$ (Hakim et al., 2010). Inhibition of seed germination and shoot and root was noticed as a result of sodium chloride treatments in rice and cabbage (Jamil et al., 2007; Sheng et al., 2011). It is considered that salinity has adverse effect of salt on plant height, root, shoot and dry matter of rice and these parameters are decreased with increasing in salt concentration (Kazemi and Eskandari, 2013; Rajakumar, 2013; Ologundudu et al., 2014). Though dissimilarity is also found where root length was increased with salt stress (Hussain et al., 2013).

Development of salt tolerant varieties has been considered as one of the strategies to increase rice production in saline prone coastal areas. Selection of salt tolerant genotypes has been carried out for over 3 decades (Flowers, 2004) and various screening methodologies used (Kuchanur et al., 2006) to screen out tolerant varieties. The present study was therefore designed to understand the mechanisms of salt tolerance in rice plant and to screen out a wide range of rice genotypes based on their tolerance though root growth parameters at different levels of salinity.

\section{Materials and Methods}

The experiment was conducted at the laboratory of the Department of Biochemistry and Molecular Biology, Bangladesh Agricultural University, Mymensingh.

\section{Study materials}

There were 127 rice genotypes comprising 120 traditional genotypes cultivated in the coastal region of Bangladesh and 7 improved varieties were used as check varieties for selection of salt tolerance. Among the genotypes 6 were previously released as moderately salt tolerant rice variety (Binadhan 8 , BR23, BRRI dhan40, BRRI dhan41, BRRI dhan47 and BRRI dhan53) and 1 was salt susceptible rice variety (Binadhan 7). 


\section{Salinity treatments}

Four salinity levels such as 0, 6, 9, 12 and $15 \mathrm{dS} \mathrm{m}^{-1}$ were maintained in the experiment. The different salinity levels were obtained by dissolving crude salt (collected from seashore) in distilled water. During dissolving salt with distilled water, the EC value was frequently measured with EC meter so that pre-fixed salinity level could be maintained. The control $(0 \mathrm{dS} \mathrm{m}$ ${ }^{1}$ ) was maintained using distilled water only.

\section{Germination test}

The study was carried out in petri dishes having $15 \mathrm{~cm}$ diameter using two layers of filter paper. Twenty five seeds were placed on filter paper and $10 \mathrm{ml}$ of treatment solutions of different salinity levels were poured in each petri dish to immerse the seeds partially for ensuring proper aeration. The petri dishes were placed on a table in the laboratory. The seeds were allowed to germinate at room temperature $\left(25 \pm 2^{\circ} \mathrm{C}\right)$. Distilled water was added to each Petri dish every day as required. The young seedlings were up-rooted 8 days after sowing (DAS). Ten randomly selected plants of each genotype from each treatment were used for data recording.

\section{Data collection}

Root length was measured from the base to top of the radical. Roots were separated from the seedlings and oven-dried at $65^{\circ} \mathrm{C}$ for a period until a constant weight was obtained. Dry weight (mg) of root of each seedling was recorded.

Percent decrease of root length and root weight, due to salinity stress was calculated using the following formula:

$$
\% \text { decrease }=\frac{\text { Traits of control treatment }- \text { Traits of salinized treatment }}{\text { Traits of control treatment }} \times 100
$$

The range of percent decrease was determined and divided into five equal groups in ascending order. The groups were chronologically scored as 1, 3, 5, 7 and 9 which represent highly tolerant, tolerant, moderately tolerant, susceptible and highly susceptible genotypes to salt stress, respectively. The grouping of rice genotypes into five distinct categories were done according to IRRI (1996).

\section{Statistical analysis}

The experiment was arranged in a completely randomized design with three replications and the data collected and calculated were analyzed using MSTAT $\mathrm{C}$ computer programs and comparison of means were tested for significance using least significant difference (LSD) test, at 0.01 level of probability.

\section{Results}

\section{Root dry weight}

Salt stress significantly reduced the root dry weight of rice genotypes (Table 1.1), but the rate of reduction was not similar across the genotypes. At salinity levels 6, 9, 12 and $15 \mathrm{dS} \mathrm{m}^{-1}$ the ranges of root dry weight were 1.2-5.0, $0.8-4.2, \quad 0.6-3.2$ and $0.2-2.4 \mathrm{mg}_{\text {plant }}{ }^{-1}$, respectively. Percent decrease of root dry weight under different levels of salinity $\left(6,9,12\right.$ and $\left.15 \mathrm{dS} \mathrm{m}^{-1}\right)$ were $-5.0-54.5 \%, 0-73.7 \%, 16.7-80 \%$ and $27.8-93.3 \%$, respectively (Table 1.2). Based on the percent reduction of root dry weight at $15 \mathrm{dS} \mathrm{m} \mathrm{m}^{-1}$ salinity, the rice genotypes were grouped into five categories and relative score values were assigned. Based on the degree of reduction of root dry weight, 8 genotypes had scored 1, 28 genotypes had 3, 40 genotypes had 5, 35 genotypes had 7 and 16 genotypes scored 9 . The genotypes possessing score 1 were Machranga, Hogla,
Pengek, Beki Balam, Bashful Balam, Tikaram, Talmugur and Rati Sail which had ability to retain root dry weight under high salinity condition. Genotypes ERI, Sada Mota Bashpai, Patnai (SW), Swarna dhan, Lal Gotal, Lambo, Tilek Kuchi, Bouari, Gota, Sadamota, Khejure Chori, Ghocca, Mondeshor, Kesa, Mohime and Kalihytra were highly susceptible to salinity and thus had least ability to sustain their root weight in saline condition.

\section{Root length}

Salt stress reduced the root length of rice significantly (Table 2). With the increasing salt concentration, the root length gradually decreased. Across the genotypes ranges of root length were $2.5-13.9,2.0-10.6,2.1-9.6$, 1.2-8.5 and 0.4-6.7 $\mathrm{cm}$ at $0,6,9,12$ and $15 \mathrm{dS} \mathrm{m}^{-1}$ salinity respectively (Table 2.2). Under control condition the mean of root length was $7.4 \mathrm{~cm}$ which was reduced by $17.1,27.9,44.1$ and $61.9 \%$ at $6,9,12$ and $15 \mathrm{dS} \mathrm{m}^{-1}$ salinity respectively. To categorize the rice genotypes on the basis of their capability to minimize the decreasing effect (on root length), tolerance index table was constructed (18-33\% decrease- score 1, highly tolerant; $34-48 \%$ decrease-3, tolerant; $49-63 \%$ decrease-5, moderately tolerant; 64-78 $\%$ decrease-7, susceptible; 79-93\% decrease- 9, highly susceptible). Based on the score value it was found that, 14 genotypes were highly tolerant, 18 genotypes were tolerant, 27 genotypes were moderately tolerant, 38 genotypes were susceptible, and 30 genotypes were highly susceptible. Genotypes Ranga Hogla, Pengek, BRRI dhan47, Jota Balam, PBRC-30, Bashful Balam, Rajshahi Balam, Arman Sardar, Khok Shail, Lal Tupi, BR23, Talmuri, BRRI dhan41 and Pokkali were classified as highly tolerant. 
Table 1. ANOVA table of root dry weight in germination stage

\begin{tabular}{|c|c|c|c|c|c|}
\hline Source & $\begin{array}{l}\text { Degrees of } \\
\text { freedom }\end{array}$ & Sum of squares & Mean square & F value & Prob \\
\hline Salinity (S) & 1 & 647.897 & 647.897 & 63238.6294 & 0.0000 \\
\hline Genotype (G) & 126 & 229.047 & 1.818 & 177.4318 & 0.0000 \\
\hline $\mathrm{S} \times \mathrm{G}$ & 126 & 94.447 & 0.750 & 73.1632 & 0.0000 \\
\hline Error & 254 & 2.602 & 0.010 & & \\
\hline Total & 507 & 973.993 & & & \\
\hline$\% \mathrm{CV}$ & 4.24 & \multirow{2}{*}{\multicolumn{2}{|c|}{$\pm \mathrm{SE}$ (genotype): 0.0506}} & & \\
\hline \multicolumn{2}{|c|}{$\pm \mathrm{SE}($ salinity): 0.0064} & & & $\pm \mathrm{SE}($ interact & 0716 \\
\hline
\end{tabular}

Table 2. Effects of salinity on root dry weight of rice genotypes at germination stage

\begin{tabular}{|c|c|c|c|c|c|c|c|c|c|c|c|}
\hline Sl. & Rice genotypes & Root d & weight & plant $^{-1}$ & & & \%Decr & ase over & ontrol & & \\
\hline & & $\begin{array}{l}0 \\
\text { dS } \text { m }^{-1}\end{array}$ & $\begin{array}{l}6 \\
\text { dS m } \\
\end{array}$ & $\begin{array}{l}9 \\
\text { dS m } \\
\end{array}$ & $\begin{array}{l}12 \\
\text { dS } ~ m^{-1}\end{array}$ & $\begin{array}{l}15 \\
\text { dS } m^{-1}\end{array}$ & $\begin{array}{l}6 \\
\text { dS m } \\
\end{array}$ & $\begin{array}{l}9 \\
\text { dS m } \\
\end{array}$ & $\begin{array}{l}12 \\
\text { dS } \mathrm{m}^{-1}\end{array}$ & $\begin{array}{l}15 \\
\text { dS } ~ m^{-1}\end{array}$ & \\
\hline 1 & Binadhan 7 & 2.0 & 1.8 & 1.2 & 1.0 & 0.4 & 10.0 & 40.0 & 50.0 & 80.0 & 7 \\
\hline 2 & Ledra binni & 3.0 & 2.6 & 1.4 & 1.2 & 1.0 & 13.3 & 53.3 & 60.0 & 66.7 & 5 \\
\hline 3 & Machranga & 2.6 & 2.2 & 2.2 & 1.6 & 1.6 & 15.4 & 15.4 & 38.5 & 38.5 & 1 \\
\hline 4 & Vushiara & 3.6 & 3.2 & 2.8 & 2.8 & 2.0 & 11.1 & 22.2 & 22.2 & 44.4 & 3 \\
\hline 5 & Dud sail & 2.0 & 1.8 & 0.8 & 0.8 & 0.4 & 10.0 & 60.0 & 60.0 & 80.0 & 7 \\
\hline 6 & Ghunshi-1 & 4.2 & 2.6 & 2.2 & 1.2 & 1.2 & 38.1 & 47.6 & 71.4 & 71.4 & 7 \\
\hline 7 & Ranga Hogla & 3.8 & 3.6 & 3.2 & 2.4 & 1.8 & 5.3 & 15.8 & 36.8 & 52.6 & 3 \\
\hline 8 & ShornaMushuri & 3.8 & 3.0 & 2.8 & 2.4 & 2.2 & 21.1 & 26.3 & 36.8 & 42.1 & 3 \\
\hline 9 & Lal 40 & 3.6 & 2.6 & 2.4 & 2.0 & 1.8 & 27.8 & 33.3 & 44.4 & 50.0 & 3 \\
\hline 10 & Gotamala & 5.0 & 4.0 & 3.0 & 1.4 & 1.2 & 20.0 & 40.0 & 72.0 & 76.0 & 7 \\
\hline 11 & Hogla & 4.2 & 3.6 & 3.2 & 3.0 & 3.0 & 14.3 & 23.8 & 28.6 & 28.6 & 1 \\
\hline 12 & Bola Balam & 3.2 & 2.4 & 1.8 & 1.6 & 1.2 & 25.0 & 43.8 & 50.0 & 62.5 & 5 \\
\hline 13 & Pengek & 4.2 & 3.6 & 3.2 & 2.8 & 2.6 & 14.3 & 23.8 & 33.3 & 38.1 & 1 \\
\hline 14 & ERI & 2.4 & 1.4 & 1.4 & 0.8 & 0.4 & 41.7 & 41.7 & 66.7 & 83.3 & 9 \\
\hline 15 & BRRI dhan40 & 2.9 & 2.4 & 1.8 & 1.4 & 0.8 & 17.2 & 37.9 & 51.7 & 72.4 & 7 \\
\hline 16 & BRRI dhan47 & 3.4 & 2.8 & 2.8 & 1.8 & 1.4 & 17.6 & 17.6 & 47.1 & 58.8 & 5 \\
\hline 17 & Jota Balam & 3.4 & 3.2 & 2.2 & 1.8 & 1.5 & 5.9 & 35.3 & 47.1 & 55.9 & 5 \\
\hline 18 & Jiradhan & 2.0 & 1.4 & 1.4 & 0.4 & 0.4 & 30.0 & 30.0 & 80.0 & 80.0 & 7 \\
\hline 19 & Patnai (FW) & 4.2 & 3.2 & 2.4 & 1.8 & 1.2 & 23.8 & 42.9 & 57.1 & 71.4 & 7 \\
\hline 20 & Sada & & & & & & & & & & \\
\hline & Bashpai & 4.4 & 4.2 & 2.4 & 1.8 & 0.6 & 4.5 & 45.5 & 59.1 & 86.4 & 9 \\
\hline 21 & Patnai (SW) & 3.6 & 2.4 & 2.2 & 0.8 & 0.6 & 33.3 & 38.9 & 77.8 & 83.3 & 9 \\
\hline 22 & Swarna Pajam & 3.4 & 2.6 & 1.4 & 1.2 & 1.1 & 23.5 & 58.8 & 64.7 & 67.6 & 5 \\
\hline 23 & Sada Gotal & 3.6 & 3.2 & 2.2 & 1.6 & 1.0 & 11.1 & 38.9 & 55.6 & 72.2 & 7 \\
\hline 24 & Swarna dhan & 3.6 & 1.8 & 1.4 & 1.0 & 0.6 & 50.0 & 61.1 & 72.2 & 83.3 & 9 \\
\hline 25 & Shaheb Kachi & 5.2 & 4.6 & 2.4 & 2.0 & 2.0 & 11.5 & 53.8 & 61.5 & 61.5 & 5 \\
\hline 26 & PBRC-30 & 2.6 & 2.4 & 2.4 & 1.6 & 1.2 & 7.7 & 7.7 & 38.5 & 53.8 & 3 \\
\hline 27 & Kongaj & 4.0 & 4.0 & 3.0 & 2.0 & 1.6 & 0.0 & 25.0 & 50.0 & 60.0 & 5 \\
\hline 28 & Lal Gotal & 3.2 & 2.2 & 1.2 & 0.8 & 0.4 & 31.3 & 62.5 & 75.0 & 87.5 & 9 \\
\hline 29 & Kathi Goccha & 4.4 & 3.4 & 3.0 & 2.4 & 2.2 & 22.7 & 31.8 & 45.5 & 50.0 & 3 \\
\hline 30 & Mala goti & 3.0 & 2.4 & 2.2 & 1.6 & 1.4 & 20.0 & 26.7 & 46.7 & 53.3 & 3 \\
\hline 31 & Lutori & 3.6 & 3.2 & 3.0 & 2.4 & 1.4 & 11.1 & 16.7 & 33.3 & 61.1 & 5 \\
\hline 32 & Mait Chal & 3.6 & 3.2 & 3.0 & 2.6 & 1.0 & 11.1 & 16.7 & 27.8 & 72.2 & 7 \\
\hline 33 & Durga Bhog & 3.0 & 2.2 & 2.0 & 1.6 & 1.4 & 26.7 & 33.3 & 46.7 & 53.3 & 3 \\
\hline 34 & Lambo & 2.8 & 1.6 & 1.6 & 1.0 & 0.4 & 42.9 & 42.9 & 64.3 & 85.7 & 9 \\
\hline 35 & Chap Shail & 3.8 & 3.4 & 3.2 & 1.4 & 1.2 & 10.5 & 15.8 & 63.2 & 68.4 & 5 \\
\hline 36 & 44 dhan & 4.2 & 2.4 & 2.6 & 1.0 & 0.8 & 42.9 & 38.1 & 76.2 & 81.0 & 7 \\
\hline 37 & Swarnalata & 3.6 & 3.2 & 2.0 & 1.6 & 1.0 & 11.1 & 44.4 & 55.6 & 72.2 & 7 \\
\hline 38 & Tilek Kuchi & 3.0 & 3.0 & 2.0 & 1.0 & 0.4 & 0.0 & 33.3 & 66.7 & 86.7 & 9 \\
\hline 39 & Dakh Shail & 2.2 & 2.0 & 1.4 & 1.2 & 1.0 & 9.1 & 36.4 & 45.5 & 54.5 & 5 \\
\hline 40 & Beki Balam & 3.2 & 3.1 & 3.2 & 2.6 & 2.2 & 3.1 & 0.0 & 18.8 & 31.3 & 1 \\
\hline 41 & Kalmilata & 4.2 & 3.2 & 2.2 & 1.6 & 1.2 & 23.8 & 47.6 & 61.9 & 71.4 & 7 \\
\hline 42 & Basmati & 4.4 & 4.1 & 2.2 & 1.6 & 1.4 & 6.8 & 50.0 & 63.6 & 68.2 & 5 \\
\hline 43 & Bouari & 4.4 & 2.0 & 2.0 & 1.4 & 0.6 & 54.5 & 54.5 & 68.2 & 86.4 & 9 \\
\hline
\end{tabular}




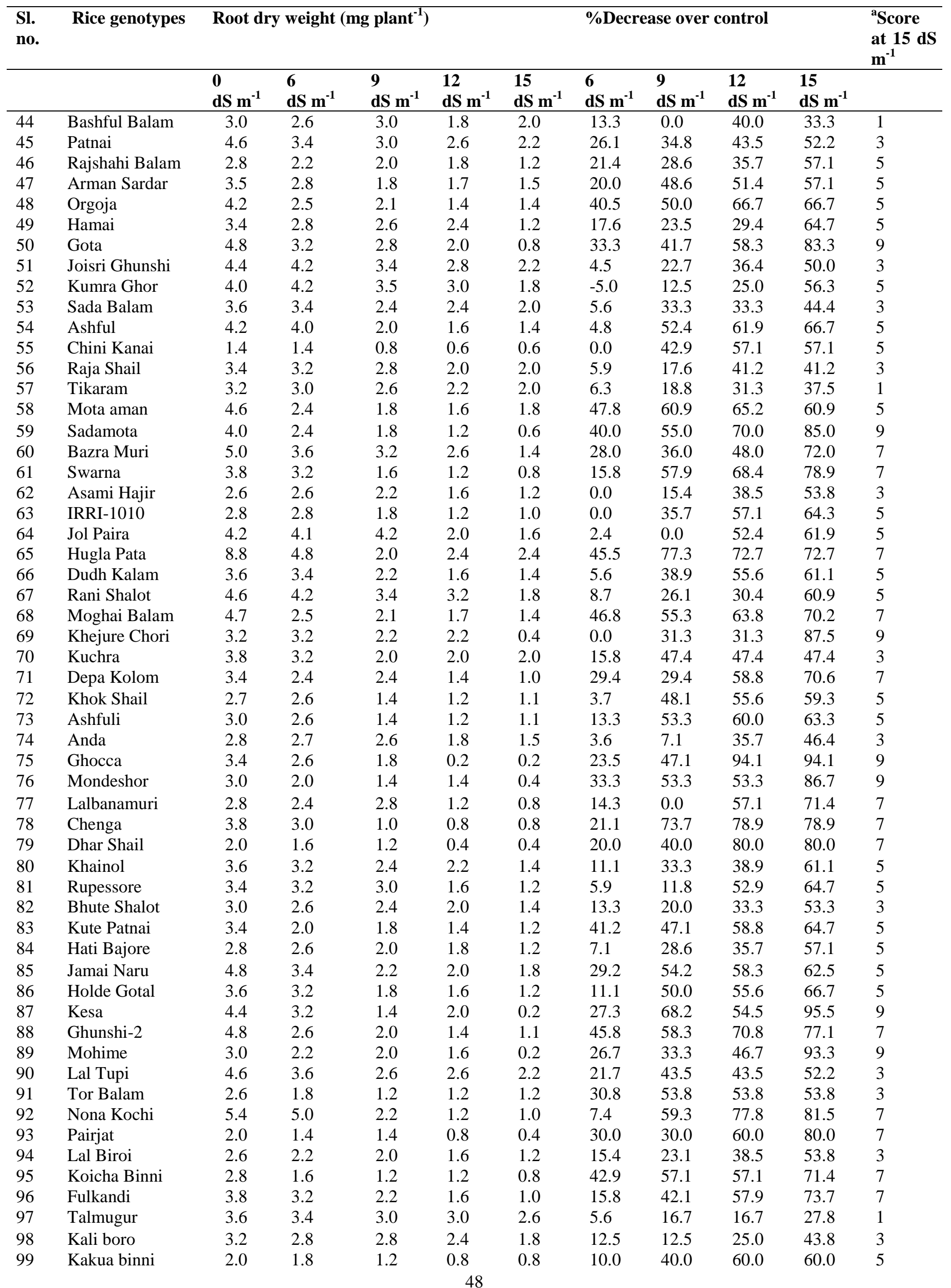




\begin{tabular}{|c|c|c|c|c|c|c|c|c|c|c|c|}
\hline \multirow{2}{*}{$\begin{array}{l}\text { Sl. } \\
\text { no. }\end{array}$} & \multirow[t]{2}{*}{ Rice genotypes } & \multicolumn{4}{|c|}{ Root dry weight $\left(\mathrm{mg} \mathrm{plant}^{-1}\right)$} & \multicolumn{5}{|c|}{ \%Decrease over control } & \multirow{2}{*}{$\begin{array}{l}{ }^{\text {a Score }} \\
\text { at } 15 \mathrm{dS} \\
\mathrm{m}^{-1}\end{array}$} \\
\hline & & $\begin{array}{l}0 \\
\mathrm{dS} \mathrm{m}^{-1}\end{array}$ & $\begin{array}{l}6 \\
\mathrm{dS} \mathrm{m}^{-1}\end{array}$ & $\begin{array}{l}9 \\
\mathrm{dS} \mathrm{m}^{-1}\end{array}$ & $\begin{array}{l}12 \\
\mathrm{dS} \mathrm{m}^{-1}\end{array}$ & $\begin{array}{l}15 \\
\mathrm{dS} \mathrm{m}^{-1}\end{array}$ & $\begin{array}{l}6 \\
\mathrm{dS} \mathrm{m}^{-1}\end{array}$ & $\begin{array}{l}9 \\
d S m^{-1}\end{array}$ & $\begin{array}{l}12 \\
\mathrm{dS} \mathrm{m}^{-1}\end{array}$ & $\begin{array}{l}15 \\
d S m^{-1}\end{array}$ & \\
\hline 100 & Pajam & 3.4 & 2.0 & 1.6 & 1.3 & 1.0 & 41.2 & 52.9 & 61.8 & 70.6 & 7 \\
\hline 101 & Nonabokra & 4.4 & 3.4 & 3.0 & 2.8 & 2.4 & 22.7 & 31.8 & 36.4 & 45.5 & 3 \\
\hline 102 & Jolkumri & 5.2 & 4.0 & 3.0 & 1.4 & 1.2 & 23.1 & 42.3 & 73.1 & 76.9 & 7 \\
\hline 103 & Kasia binni & 3.2 & 2.8 & 2.6 & 2.4 & 1.0 & 12.5 & 18.8 & 25.0 & 68.8 & 7 \\
\hline 104 & Gigoj & 3.6 & 3.0 & 2.4 & 1.8 & 1.4 & 16.7 & 33.3 & 50.0 & 61.1 & 5 \\
\hline 105 & Rati Sail & 3.0 & 2.0 & 2.0 & 2.0 & 1.8 & 33.3 & 33.3 & 33.3 & 40.0 & 1 \\
\hline 106 & Sakal Mukhi & 4.5 & 3.2 & 2.4 & 1.8 & 1.2 & 28.9 & 46.7 & 60.0 & 73.3 & 7 \\
\hline 107 & Golapi & 3.9 & 3.2 & 2.8 & 2.6 & 2.0 & 17.9 & 28.2 & 33.3 & 48.7 & 3 \\
\hline 108 & Malshira & 3.4 & 3.2 & 2.2 & 1.8 & 1.1 & 5.9 & 35.3 & 47.1 & 67.6 & 5 \\
\hline 109 & Pankhiraj & 3.4 & 2.8 & 2.4 & 1.6 & 1.4 & 17.6 & 29.4 & 52.9 & 58.8 & 5 \\
\hline 110 & Jongliboro & 2.0 & 1.8 & 1.2 & 1.2 & 0.8 & 10.0 & 40.0 & 40.0 & 60.0 & 5 \\
\hline 111 & Nunia & 1.2 & 1.2 & 1.0 & 1.0 & 0.6 & 0.0 & 16.7 & 16.7 & 50.0 & 3 \\
\hline 112 & Nunia-1 & 1.6 & 1.0 & 0.8 & 0.8 & 0.4 & 37.5 & 50.0 & 50.0 & 75.0 & 7 \\
\hline 113 & Minikit & 3.2 & 2.0 & 1.8 & 1.6 & 0.8 & 37.5 & 43.8 & 50.0 & 75.0 & 7 \\
\hline 114 & BR23 & 3.0 & 3.0 & 2.3 & 1.9 & 1.4 & 0.0 & 23.3 & 36.7 & 53.3 & 3 \\
\hline 115 & Kalihytra & 3.0 & 1.4 & 2.4 & 1.4 & 0.2 & 53.3 & 20.0 & 53.3 & 93.3 & 9 \\
\hline 116 & Khato Komro & 3.2 & 2.4 & 2.2 & 0.8 & 0.6 & 25.0 & 31.3 & 75.0 & 81.3 & 7 \\
\hline 117 & Talmuri & 5.0 & 4.0 & 3.4 & 2.6 & 2.2 & 20.0 & 32.0 & 48.0 & 56.0 & 5 \\
\hline 118 & Bekas & 3.2 & 2.2 & 1.8 & 1.4 & 0.8 & 31.3 & 43.8 & 56.3 & 75.0 & 7 \\
\hline 119 & Patnai Balam & 4.0 & 2.4 & 2.2 & 2.0 & 1.8 & 40.0 & 45.0 & 50.0 & 55.0 & 5 \\
\hline 120 & Khesrail & 3.4 & 3.3 & 3.0 & 2.2 & 1.8 & 2.9 & 11.8 & 35.3 & 47.1 & 3 \\
\hline 121 & Kajal Sail & 4.0 & 4.0 & 3.8 & 2.6 & 1.6 & 0.0 & 5.0 & 35.0 & 60.0 & 5 \\
\hline 122 & Binadhan 8 & 4.2 & 3.4 & 2.6 & 2.2 & 1.8 & 19.0 & 38.1 & 47.6 & 57.1 & 5 \\
\hline 123 & BRRI dhan41 & 2.5 & 2.2 & 2.1 & 1.7 & 1.2 & 12.0 & 16.0 & 32.0 & 52.0 & 3 \\
\hline 124 & Kuti Patnai & 2.6 & 2.4 & 2.4 & 1.6 & 1.2 & 7.7 & 7.7 & 38.5 & 53.8 & 3 \\
\hline 125 & Dorkumur & 3.4 & 1.6 & 1.4 & 1.2 & 1.0 & 52.9 & 58.8 & 64.7 & 70.6 & 7 \\
\hline 126 & BRRI dhan53 & 2.4 & 2.2 & 2.0 & 0.8 & 0.7 & 8.3 & 16.7 & 66.7 & 70.8 & 7 \\
\hline 127 & Pokkali & 3.4 & 3.4 & 2.6 & 2.2 & 2.0 & 0.0 & 23.5 & 35.3 & 41.2 & 3 \\
\hline & Mean & 3.5 & 2.8 & 2.2 & 1.7 & 1.3 & 20.0 & 36.8 & 51.8 & 64.3 & \\
\hline
\end{tabular}

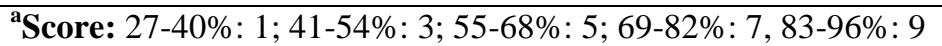

Table 3. ANOVA table of root length in germination stage

\begin{tabular}{|c|c|c|c|c|c|}
\hline Source & $\begin{array}{l}\text { Degrees of } \\
\text { freedom }\end{array}$ & Sum of squares & Mean square & F value & Prob \\
\hline Salinity (S) & 1 & 2819.830 & 2819.830 & 70736.6497 & 0.0000 \\
\hline Genotype (G) & 126 & 966.203 & 7.668 & 192.3620 & 0.0000 \\
\hline $\mathrm{S} \times \mathrm{G}$ & 126 & 684.439 & 5.432 & 136.2654 & 0.0000 \\
\hline Error & 254 & 10.125 & 0.040 & & \\
\hline Total & 507 & 4480.598 & & & \\
\hline$\% \mathrm{CV}$ & 3.99 & \multirow{2}{*}{\multicolumn{2}{|c|}{ \pm SE(genotype): 0.0998}} & & \\
\hline \multicolumn{2}{|c|}{$\pm \mathrm{SE}$ (salinity): 0.0125} & & & \multicolumn{2}{|c|}{$\pm \mathrm{SE}($ interaction $): 0.1412$} \\
\hline
\end{tabular}

Table 4. Effects of salinity on the root length of rice genotypes at germination stage

\begin{tabular}{|c|c|c|c|c|c|c|c|c|c|c|c|}
\hline \multirow{2}{*}{$\begin{array}{l}\text { Sl. } \\
\text { no. }\end{array}$} & \multirow[t]{2}{*}{ Rice genotypes } & \multicolumn{4}{|c|}{ Root length (cm plant ${ }^{-1)}$} & \multicolumn{5}{|c|}{ \%Decrease over control } & \multirow{2}{*}{$\begin{array}{l}{ }^{\text {a Score }} \\
\text { at } 15 \mathrm{dS} \\
\mathrm{m}^{-1}\end{array}$} \\
\hline & & $\begin{array}{l}0 \\
\text { dS m } \text { m }^{-1}\end{array}$ & $\begin{array}{l}6 \\
\mathrm{dS} \mathrm{m}^{-1}\end{array}$ & $\begin{array}{l}9 \\
\mathrm{dS} \mathrm{m}^{-1}\end{array}$ & $\begin{array}{l}12 \\
\mathrm{dS} \mathrm{m}^{-1}\end{array}$ & $\begin{array}{l}15 \\
\mathrm{dS} \mathrm{m}^{-1}\end{array}$ & $\begin{array}{l}6 \\
\mathrm{dS} \mathrm{m}^{-1}\end{array}$ & $\begin{array}{l}9 \\
\mathrm{dS} \mathrm{m}^{-1}\end{array}$ & $\begin{array}{l}12 \\
\mathrm{dS} \mathrm{m}^{-1}\end{array}$ & $\begin{array}{l}15 \\
\mathrm{dS} \mathrm{m}^{-1}\end{array}$ & \\
\hline 1 & Binadhan 7 & 3.5 & 3.4 & 3.1 & 2.8 & 1.8 & 2.9 & 11.4 & 20.0 & 48.6 & 5 \\
\hline 2 & Ledra binni & 5.0 & 4.0 & 4.2 & 3.2 & 1.6 & 20.0 & 16.0 & 36.0 & 68.0 & 7 \\
\hline 3 & Machranga & 6.8 & 4.7 & 3.2 & 2.2 & 1.5 & 30.9 & 52.9 & 67.6 & 77.9 & 7 \\
\hline 4 & Vushiara & 6.5 & 6.4 & 6.3 & 4.9 & 3.0 & 1.5 & 3.1 & 24.6 & 53.8 & 5 \\
\hline 5 & Dud sail & 9.0 & 8.0 & 5.5 & 5.0 & 2.3 & 11.1 & 38.9 & 44.4 & 74.4 & 7 \\
\hline 6 & Ghunshi-1 & 5.5 & 4.8 & 4.1 & 3.8 & 1.9 & 12.7 & 25.5 & 30.9 & 65.5 & 7 \\
\hline 7 & Ranga Hogla & 5.2 & 4.8 & 4.8 & 4.6 & 4.0 & 7.7 & 7.7 & 11.5 & 23.1 & 1 \\
\hline 8 & ShornaMushuri & 10.1 & 6.5 & 5.2 & 3.2 & 1.7 & 35.6 & 48.5 & 68.3 & 83.2 & 9 \\
\hline 9 & Lal 40 & 7.8 & 7.5 & 7.2 & 6.6 & 4.1 & 3.8 & 7.7 & 15.4 & 47.4 & 3 \\
\hline
\end{tabular}




\begin{tabular}{|c|c|c|c|c|c|c|c|c|c|c|c|}
\hline \multirow{2}{*}{$\begin{array}{l}\text { SI. } \\
\text { no. }\end{array}$} & \multirow[t]{2}{*}{ Rice genotypes } & \multicolumn{3}{|c|}{ Root length (cm plant ${ }^{-1)}$} & \multirow[b]{2}{*}{$\begin{array}{l}12 \\
\mathrm{dS} \mathrm{m}^{-1}\end{array}$} & \multicolumn{5}{|c|}{ \%Decrease over control } & \multirow{2}{*}{$\begin{array}{l}{ }^{\mathrm{a}} \text { Score } \\
\text { at } 15 \mathrm{dS} \\
\mathrm{m}^{-1}\end{array}$} \\
\hline & & $\begin{array}{l}0 \\
\mathrm{dS} \mathrm{m}^{-1}\end{array}$ & $\begin{array}{l}6 \\
\mathrm{dS} \mathrm{m}^{-1}\end{array}$ & $\begin{array}{l}9 \\
\mathrm{dS} \mathrm{m}^{-1}\end{array}$ & & $\begin{array}{l}15 \\
\mathrm{dS} \mathrm{m}^{-1}\end{array}$ & $\begin{array}{l}6 \\
\mathrm{dS} \mathrm{m}^{-1}\end{array}$ & $\begin{array}{l}9 \\
\mathrm{dS} \mathrm{m}^{-1}\end{array}$ & $\begin{array}{l}12 \\
\mathrm{dS} \mathrm{m}^{-1}\end{array}$ & $\begin{array}{l}15 \\
d S ~ m^{-1}\end{array}$ & \\
\hline 10 & Gotamala & 6.5 & 5.2 & 3.8 & 3.4 & 3.0 & 20.0 & 42.3 & 48.5 & 54.6 & 5 \\
\hline 11 & Hogla & 4.1 & 3.6 & 3.2 & 2.8 & 2.6 & 12.2 & 22.0 & 31.7 & 36.6 & 3 \\
\hline 12 & Bola Balam & 5.4 & 4.3 & 3.1 & 1.5 & 1.8 & 20.4 & 42.6 & 72.2 & 66.7 & 7 \\
\hline 13 & Pengek & 8.1 & 7.4 & 7.0 & 6.6 & 5.7 & 8.6 & 13.6 & 18.5 & 29.6 & 1 \\
\hline 14 & ERI & 2.5 & 2.0 & 2.4 & 2.3 & 1.1 & 20.0 & 5.0 & 8.0 & 55.0 & 5 \\
\hline 15 & BRRI dhan40 & 5.9 & 5.4 & 4.3 & 3.7 & 3.6 & 8.5 & 27.1 & 38.1 & 39.0 & 3 \\
\hline 16 & BRRI dhan47 & 5.9 & 5.8 & 5.2 & 4.9 & 4.8 & 1.7 & 12.5 & 17.6 & 19.0 & 1 \\
\hline 17 & Jota Balam & 5.2 & 4.7 & 4.7 & 4.4 & 3.8 & 9.6 & 9.6 & 15.4 & 26.9 & 1 \\
\hline 18 & Jiradhan & 7.9 & 6.9 & 4.6 & 5.8 & 3.9 & 13.2 & 41.8 & 26.6 & 50.6 & 5 \\
\hline 19 & Patnai (FW) & 13.9 & 9.8 & 4.3 & 4.2 & 2.7 & 29.5 & 69.1 & 69.8 & 80.6 & 9 \\
\hline \multirow[t]{2}{*}{20} & Sada Mota & & & & & & & & & & 3 \\
\hline & Bashpai & 6.4 & 6.0 & 4.2 & 3.8 & 3.5 & 6.3 & 34.1 & 41.1 & 45.3 & \\
\hline 21 & Patnai (SW) & 8.0 & 6.8 & 6.0 & 5.8 & 3.1 & 15.0 & 25.0 & 27.5 & 61.3 & 5 \\
\hline 22 & Swarna Pajam & 6.2 & 3.5 & 3.6 & 2.6 & 0.8 & 43.5 & 41.9 & 58.1 & 87.1 & 9 \\
\hline 23 & Sada Gotal & 7.9 & 6.1 & 5.8 & 4.9 & 2.6 & 22.8 & 26.6 & 38.0 & 66.7 & 7 \\
\hline 24 & Swarna dhan & 8.2 & 7.4 & 5.1 & 2.9 & 3.3 & 9.5 & 37.8 & 64.6 & 60.4 & 5 \\
\hline 25 & Shaheb Kachi & 7.3 & 6.2 & 4.9 & 5.0 & 3.1 & 15.1 & 32.9 & 31.5 & 57.5 & 5 \\
\hline 26 & PBRC-30 & 8.2 & 7.4 & 6.6 & 6.0 & 5.8 & 9.8 & 19.5 & 26.8 & 29.3 & 1 \\
\hline 27 & Kongaj & 8.8 & 6.0 & 6.2 & 4.2 & 4.1 & 31.8 & 29.5 & 52.3 & 53.4 & 5 \\
\hline 28 & Lal Gotal & 6.6 & 6.3 & 5.3 & 4.0 & 1.8 & 4.5 & 19.7 & 39.4 & 72.7 & 7 \\
\hline 29 & Kathi Goccha & 6.6 & 6.5 & 5.7 & 3.5 & 2.9 & 1.5 & 13.6 & 47.0 & 56.1 & 5 \\
\hline 30 & Mala goti & 6.5 & 4.7 & 4.6 & 3.4 & 3.3 & 27.7 & 29.2 & 47.7 & 49.2 & 5 \\
\hline 31 & Lutori & 9.6 & 8.0 & 6.4 & 4.7 & 2.2 & 16.7 & 33.3 & 51.0 & 77.3 & 7 \\
\hline 32 & Mait Chal & 5.6 & 5.5 & 5.4 & 4.5 & 2.6 & 1.8 & 3.6 & 19.6 & 53.6 & 5 \\
\hline 33 & Durga Bhog & 7.4 & 5.5 & 5.1 & 3.4 & 1.1 & 25.7 & 31.1 & 54.1 & 85.1 & 9 \\
\hline 34 & Lambo & 6.8 & 5.0 & 4.0 & 3.4 & 1.4 & 26.5 & 41.2 & 50.0 & 79.4 & 9 \\
\hline 35 & Chap Shail & 7.8 & 6.3 & 5.5 & 3.3 & 1.7 & 19.4 & 29.7 & 57.5 & 77.7 & 7 \\
\hline 36 & 44 dhan & 7.2 & 4.8 & 4.7 & 4.6 & 2.0 & 33.3 & 34.7 & 36.1 & 72.2 & 7 \\
\hline 37 & Swarnalata & 9.8 & 8.9 & 7.8 & 6.0 & 3.3 & 9.2 & 20.4 & 38.8 & 66.3 & 7 \\
\hline 38 & Tilek Kuchi & 11.3 & 6.5 & 5.7 & 6.1 & 1.5 & 42.5 & 49.6 & 46.0 & 86.7 & 9 \\
\hline 39 & Dakh Shail & 7.0 & 6.6 & 6.0 & 5.8 & 3.3 & 5.7 & 14.3 & 17.1 & 52.9 & 5 \\
\hline 40 & Beki Balam & 5.2 & 5.1 & 4.8 & 3.6 & 3.0 & 1.9 & 7.7 & 30.8 & 42.3 & 3 \\
\hline 41 & Kalmilata & 8.7 & 7.8 & 6.8 & 2.8 & 2.0 & 10.3 & 21.8 & 67.8 & 77.0 & 7 \\
\hline 42 & Basmati & 4.5 & 3.0 & 2.9 & 2.0 & 0.8 & 33.3 & 35.6 & 55.6 & 82.2 & 9 \\
\hline 43 & Bouari & 9.3 & 8.8 & 5.3 & 3.8 & 1.6 & 5.4 & 43.4 & 59.1 & 82.8 & 9 \\
\hline 44 & Bashful Balam & 6.5 & 6.0 & 5.0 & 5.2 & 4.8 & 7.7 & 23.1 & 20.0 & 26.2 & 1 \\
\hline 45 & Patnai & 6.0 & 5.8 & 4.1 & 3.6 & 3.4 & 3.3 & 31.7 & 40.0 & 43.3 & 3 \\
\hline \multirow[t]{2}{*}{46} & Rajshahi Balam & & & & & & & & & & \\
\hline & & 5.1 & 5.0 & 4.9 & 4.1 & 3.7 & 2.0 & 3.9 & 19.6 & 27.5 & 1 \\
\hline 47 & Arman Sardar & 6.0 & 5.7 & 5.6 & 4.5 & 4.0 & 5.0 & 6.7 & 25.0 & 33.3 & 1 \\
\hline 48 & Orgoja & 6.8 & 6.2 & 5.3 & 4.0 & 2.3 & 8.8 & 22.1 & 40.6 & 66.2 & 7 \\
\hline 49 & Hamai & 9.4 & 9.2 & 3.8 & 4.4 & 3.8 & 2.1 & 59.6 & 53.2 & 59.6 & 5 \\
\hline 50 & Gota & 6.5 & 4.6 & 6.0 & 3.7 & 2.3 & 29.2 & 7.7 & 43.1 & 64.6 & 7 \\
\hline 51 & Joisri Ghunshi & 8.0 & 4.8 & 3.8 & 3.3 & 2.8 & 39.9 & 52.5 & 58.8 & 65.0 & 7 \\
\hline 52 & Kumra Ghor & 6.8 & 5.8 & 4.6 & 3.1 & 1.9 & 14.7 & 32.1 & 54.4 & 72.1 & 7 \\
\hline 53 & Sada Balam & 10.8 & 7.6 & 6.1 & 6.1 & 1.0 & 29.6 & 43.5 & 43.5 & 90.7 & 9 \\
\hline 54 & Ashful & 7.6 & 6.0 & 5.1 & 4.0 & 3.1 & 21.1 & 32.9 & 47.4 & 59.2 & 5 \\
\hline 55 & Chini Kanai & 7.3 & 6.4 & 4.8 & 3.0 & 1.8 & 12.3 & 34.2 & 58.9 & 75.3 & 7 \\
\hline 56 & Raja Shail & 7.7 & 7.6 & 6.8 & 4.6 & 1.9 & 0.5 & 11.5 & 40.5 & 74.7 & 7 \\
\hline 57 & Tikaram & 9.6 & 7.8 & 8.1 & 4.4 & 2.5 & 19.2 & 15.8 & 54.4 & 74.0 & 7 \\
\hline 58 & Mota Aman & 4.7 & 4.5 & 4.5 & 2.3 & 1.2 & 4.3 & 4.3 & 51.1 & 74.5 & 7 \\
\hline 59 & Sadamota & 6.1 & 4.2 & 3.7 & 2.0 & 1.9 & 31.1 & 39.3 & 67.2 & 68.9 & 7 \\
\hline 60 & Bazra Muri & 5.6 & 5.1 & 4.6 & 2.6 & 1.2 & 8.9 & 17.9 & 53.6 & 78.6 & 9 \\
\hline 61 & Swarna & 6.6 & 4.7 & 3.2 & 2.2 & 1.5 & 28.8 & 51.5 & 66.7 & 77.3 & 7 \\
\hline 62 & Asami Hajir & 10.5 & 9.0 & 5.3 & 4.0 & 5.0 & 13.9 & 49.2 & 61.9 & 52.4 & 5 \\
\hline 63 & IRRI-1010 & 10.5 & 5.1 & 5.2 & 3.4 & 3.7 & 51.4 & 50.5 & 67.9 & 64.8 & 7 \\
\hline 64 & Jol Paira & 7.2 & 6.2 & 7.0 & 6.6 & 3.8 & 13.9 & 2.8 & 8.3 & 47.8 & 3 \\
\hline
\end{tabular}




\begin{tabular}{|c|c|c|c|c|c|c|c|c|c|c|c|}
\hline \multirow{2}{*}{$\begin{array}{l}\text { Sl. } \\
\text { no. }\end{array}$} & \multirow[t]{2}{*}{ Rice genotypes } & \multicolumn{3}{|c|}{ Root length (cm plant ${ }^{-1)}$} & \multirow[b]{2}{*}{$\begin{array}{l}12 \\
\mathrm{dS} \mathrm{m}^{-1}\end{array}$} & \multicolumn{5}{|c|}{ \% Decrease over control } & \multirow{2}{*}{$\begin{array}{l}{ }^{\text {a Score }} \\
\text { at } 15 \mathrm{dS} \\
\mathrm{m}^{-1}\end{array}$} \\
\hline & & $\begin{array}{l}0 \\
\mathrm{dS} \mathrm{m}^{-1}\end{array}$ & $\begin{array}{l}6 \\
\mathrm{dS} \mathrm{m}^{-1}\end{array}$ & $\begin{array}{l}9 \\
\mathrm{dS} \mathrm{m}^{-1}\end{array}$ & & $\begin{array}{l}15 \\
\mathrm{dS} \mathrm{m}^{-1}\end{array}$ & $\begin{array}{l}6 \\
\text { dS m } \\
\end{array}$ & $\begin{array}{l}9 \\
\mathrm{dS} \mathrm{m}^{-1}\end{array}$ & $\begin{array}{l}12 \\
\mathrm{dS} \mathrm{m}^{-1}\end{array}$ & $\begin{array}{l}15 \\
\text { dS } ~ m^{-1}\end{array}$ & \\
\hline 65 & Hugla Pata & 7.2 & 6.9 & 4.6 & 5.8 & 3.9 & 4.7 & 36.1 & 19.4 & 45.8 & 3 \\
\hline 66 & Dudh Kalam & 9.8 & 7.3 & 6.2 & 2.9 & 2.5 & 25.8 & 37.0 & 70.5 & 74.2 & 7 \\
\hline 67 & Rani Shalot & 9.9 & 8.4 & 7.2 & 6.2 & 5.8 & 15.2 & 27.3 & 37.4 & 41.4 & 3 \\
\hline 68 & Moghai Balam & 6.8 & 5.8 & 4.6 & 3.1 & 1.9 & 14.7 & 32.1 & 54.4 & 72.1 & 7 \\
\hline 69 & Khejure Chori & 9.2 & 8.2 & 6.4 & 4.7 & 2.2 & 10.9 & 30.4 & 48.9 & 76.3 & 7 \\
\hline 70 & Kuchra & 7.9 & 6.0 & 6.8 & 4.8 & 3.1 & 24.1 & 13.9 & 39.2 & 60.8 & 5 \\
\hline 71 & Depa Kolom & 9.4 & 8.8 & 8.5 & 6.9 & 4.8 & 6.4 & 9.6 & 26.6 & 48.9 & 5 \\
\hline 72 & Khok Shail & 7.0 & 6.7 & 6.0 & 5.8 & 5.0 & 4.3 & 14.3 & 17.1 & 28.6 & 1 \\
\hline 73 & Ashfuli & 9.0 & 8.0 & 5.5 & 5.0 & 2.3 & 11.1 & 38.9 & 44.4 & 74.4 & 7 \\
\hline 74 & Anda & 12.8 & 6.8 & 5.0 & 3.2 & 2.4 & 46.9 & 61.1 & 74.7 & 81.1 & 9 \\
\hline 75 & Ghocca & 10.2 & 6.8 & 6.1 & 4.2 & 2.0 & 33.3 & 40.2 & 58.8 & 80.4 & 9 \\
\hline 76 & Mondeshor & 8.0 & 6.2 & 4.8 & 3.6 & 1.6 & 22.5 & 40.0 & 55.0 & 80.0 & 9 \\
\hline 77 & Lalbanamuri & 10.0 & 4.5 & 6.0 & 2.8 & 1.0 & 54.5 & 40.1 & 71.9 & 90.0 & 9 \\
\hline 78 & Chenga & 6.7 & 5.2 & 6.3 & 1.6 & 0.8 & 21.8 & 6.0 & 76.1 & 88.1 & 9 \\
\hline 79 & Dhar Shail & 4.1 & 3.1 & 2.4 & 1.7 & 0.4 & 24.4 & 41.5 & 58.5 & 90.2 & 9 \\
\hline 80 & Khainol & 7.4 & 6.5 & 5.2 & 4.4 & 2.0 & 12.2 & 29.7 & 40.5 & 73.0 & 7 \\
\hline 81 & Rupessore & 8.3 & 6.3 & 5.5 & 3.3 & 1.7 & 24.1 & 33.7 & 60.0 & 79.0 & 9 \\
\hline 82 & Bhute Shalot & 6.5 & 6.4 & 5.8 & 4.3 & 3.9 & 1.5 & 10.8 & 33.8 & 40.0 & 3 \\
\hline 83 & Kute Patnai & 9.2 & 5.1 & 7.0 & 4.9 & 3.3 & 44.6 & 24.3 & 46.7 & 64.1 & 7 \\
\hline 84 & Hati Bajore & 7.0 & 7.0 & 6.0 & 1.8 & 1.5 & 0.0 & 14.3 & 74.3 & 78.6 & 9 \\
\hline 85 & Jamai Naru & 5.8 & 5.4 & 5.8 & 4.4 & 3.1 & 6.9 & 0.9 & 24.1 & 46.6 & 3 \\
\hline 86 & Holde Gotal & 7.0 & 7.0 & 5.2 & 1.2 & 1.2 & 0.0 & 25.7 & 82.9 & 82.9 & 9 \\
\hline 87 & Kesa & 10.3 & 8.3 & 8.2 & 5.3 & 1.6 & 19.4 & 20.4 & 48.5 & 84.5 & 9 \\
\hline 88 & Ghunshi-2 & 5.7 & 4.6 & 4.3 & 3.2 & 1.5 & 19.3 & 24.6 & 43.9 & 73.7 & 7 \\
\hline 89 & Mohime & 10.6 & 9.6 & 7.4 & 5.6 & 1.9 & 9.8 & 30.5 & 47.4 & 82.1 & 9 \\
\hline 90 & Lal Tupi & 2.7 & 2.6 & 2.3 & 2.5 & 1.8 & 3.7 & 14.8 & 7.4 & 33.3 & 1 \\
\hline 91 & Tor Balam & 8.6 & 6.2 & 6.9 & 4.1 & 2.4 & 27.9 & 19.8 & 52.3 & 72.1 & 7 \\
\hline 92 & Nona Kochi & 10.4 & 8.3 & 5.3 & 2.5 & 1.8 & 20.5 & 48.9 & 76.1 & 82.8 & 9 \\
\hline 93 & Pairjat & 7.2 & 6.2 & 5.6 & 5.2 & 3.0 & 13.3 & 22.2 & 27.8 & 58.3 & 5 \\
\hline 94 & Lal Biroi & 5.4 & 3.5 & 3.6 & 2.6 & 0.8 & 35.2 & 33.3 & 51.9 & 85.2 & 9 \\
\hline 95 & Koicha Binni & 5.1 & 5.0 & 4.3 & 1.9 & 0.8 & 2.0 & 15.7 & 62.7 & 84.3 & 9 \\
\hline 96 & Fulkandi & 6.9 & 5.4 & 4.3 & 3.7 & 2.6 & 21.7 & 37.7 & 47.1 & 62.3 & 5 \\
\hline 97 & Talmugur & 5.0 & 4.2 & 3.6 & 3.3 & 2.9 & 16.0 & 28.0 & 34.0 & 42.0 & 3 \\
\hline 98 & Kali boro & 10.8 & 8.4 & 5.0 & 4.0 & 2.5 & 22.2 & 53.7 & 63.0 & 76.9 & 7 \\
\hline 99 & Kakua binni & 4.0 & 3.0 & 2.9 & 2.0 & 0.8 & 25.0 & 27.5 & 50.0 & 80.0 & 9 \\
\hline 100 & Pajam & 8.6 & 7.7 & 7.1 & 7.0 & 4.1 & 10.5 & 17.4 & 18.6 & 52.3 & 5 \\
\hline 101 & Nonabokra & 3.8 & 3.4 & 2.8 & 2.9 & 2.5 & 10.5 & 26.3 & 23.7 & 34.2 & 3 \\
\hline 102 & Jolkumri & 6.3 & 4.3 & 4.9 & 3.9 & 1.7 & 31.7 & 22.2 & 38.1 & 73.0 & 7 \\
\hline 103 & Kasia binni & 7.1 & 5.4 & 4.0 & 3.3 & 1.2 & 23.9 & 43.7 & 53.5 & 83.1 & 9 \\
\hline 104 & Gigoj & 11.1 & 5.4 & 3.5 & 2.6 & 1.4 & 51.4 & 68.5 & 76.6 & 87.4 & 9 \\
\hline 105 & Rati Sail & 10.8 & 10.6 & 9.6 & 7.8 & 4.3 & 1.9 & 11.5 & 27.8 & 60.6 & 5 \\
\hline 106 & Sakal Mukhi & 8.3 & 7.5 & 7.2 & 6.6 & 3.1 & 9.6 & 13.3 & 20.5 & 62.7 & 5 \\
\hline 107 & Golapi & 8.9 & 7.4 & 5.1 & 2.9 & 3.1 & 16.6 & 42.7 & 67.4 & 65.2 & 7 \\
\hline 108 & Malshira & 8.0 & 6.2 & 4.8 & 3.6 & 1.8 & 22.5 & 40.0 & 55.0 & 77.5 & 7 \\
\hline 109 & Pankhiraj & 10.5 & 8.8 & 8.6 & 8.5 & 6.7 & 16.2 & 18.1 & 19.0 & 36.2 & 3 \\
\hline 110 & Jongliboro & 8.4 & 7.5 & 6.5 & 4.3 & 4.1 & 10.7 & 22.6 & 48.8 & 51.2 & 5 \\
\hline 111 & Nunia & 4.7 & 3.6 & 2.4 & 1.9 & 1.0 & 21.9 & 47.6 & 59.2 & 78.5 & 9 \\
\hline 112 & Nunia-1 & 5.2 & 4.6 & 3.3 & 2.4 & 1.5 & 11.5 & 36.5 & 53.8 & 71.2 & 7 \\
\hline 113 & Minikit & 3.1 & 2.1 & 2.1 & 2.0 & 1.3 & 32.3 & 32.3 & 35.5 & 58.1 & 5 \\
\hline 114 & BR23 & 7.3 & 7.3 & 7.2 & 7.1 & 6.0 & 0.0 & 2.1 & 2.7 & 18.5 & 1 \\
\hline 115 & Kalihytra & 11.1 & 8.6 & 8.0 & 4.3 & 1.3 & 22.1 & 27.3 & 61.1 & 88.7 & 9 \\
\hline 116 & Khato Komro & 7.4 & 6.5 & 5.7 & 1.5 & 2.8 & 12.2 & 23.0 & 79.7 & 62.2 & 5 \\
\hline 117 & Talmuri & 3.4 & 3.4 & 2.9 & 3.1 & 2.5 & 0.0 & 14.7 & 8.8 & 26.5 & 1 \\
\hline 118 & Bekas & 6.4 & 4.2 & 3.7 & 2.0 & 1.9 & 34.4 & 42.2 & 68.8 & 70.3 & 7 \\
\hline 119 & Patnai Balam & 6.5 & 4.9 & 4.7 & 3.8 & 3.1 & 24.6 & 27.7 & 41.5 & 52.3 & 5 \\
\hline 120 & Khesrail & 6.2 & 5.7 & 5.4 & 4.0 & 3.9 & 8.1 & 12.9 & 35.5 & 37.1 & 3 \\
\hline
\end{tabular}




\begin{tabular}{|c|c|c|c|c|c|c|c|c|c|c|c|}
\hline \multirow{2}{*}{$\begin{array}{l}\text { Sl. } \\
\text { no. }\end{array}$} & \multirow[t]{2}{*}{ Rice genotypes } & \multicolumn{4}{|c|}{ Root length (cm plant $^{-1)}$} & \multicolumn{5}{|c|}{ \% Decrease over control } & \multirow{2}{*}{$\begin{array}{l}{ }^{\text {a}} \text { Score } \\
\text { at } 15 \mathrm{dS} \\
\mathrm{m}^{-1}\end{array}$} \\
\hline & & $\begin{array}{l}0 \\
d S m^{-1}\end{array}$ & $\begin{array}{l}6 \\
d S m^{-1}\end{array}$ & $\begin{array}{l}9 \\
\mathrm{dS} \mathrm{m}^{-1}\end{array}$ & $\begin{array}{l}12 \\
\mathrm{dS} \mathrm{m}^{-1}\end{array}$ & $\begin{array}{l}15 \\
d S m^{-1}\end{array}$ & $\begin{array}{l}6 \\
\mathrm{dS} \mathrm{m}^{-1}\end{array}$ & $\begin{array}{l}9 \\
\mathrm{dS} \mathrm{m}^{-1}\end{array}$ & $\begin{array}{l}12 \\
\mathrm{dS} \mathrm{m}^{-1}\end{array}$ & $\begin{array}{l}15 \\
d S m^{-1}\end{array}$ & \\
\hline 121 & Kajal Sail & 4.5 & 3.8 & 3.6 & 3.1 & 2.7 & 15.6 & 20.0 & 31.1 & 40.0 & 3 \\
\hline 122 & Binadhan 8 & 3.8 & 3.5 & 2.8 & 2.3 & 2.0 & 7.9 & 26.3 & 39.5 & 47.4 & 3 \\
\hline 123 & BRRI dhan41 & 7.5 & 6.1 & 6.4 & 6.0 & 5.9 & 18.1 & 14.1 & 19.5 & 21.5 & 1 \\
\hline 124 & Kuti Patnai & 7.2 & 6.1 & 2.8 & 2.8 & 2.0 & 15.3 & 61.1 & 61.1 & 72.2 & 7 \\
\hline 125 & Dorkumur & 13.3 & 7.6 & 8.6 & 4.5 & 2.7 & 42.6 & 35.1 & 66.0 & 79.6 & 9 \\
\hline 126 & BRRI dhan53 & 5.5 & 5.2 & 3.8 & 3.4 & 3.0 & 5.5 & 31.8 & 39.1 & 46.4 & 3 \\
\hline 127 & Pokkali & 7.6 & 7.0 & 6.4 & 5.6 & 5.4 & 7.9 & 15.8 & 26.3 & 28.9 & 1 \\
\hline & Mean & 7.4 & 6.0 & 5.2 & 4.0 & 2.7 & 17.1 & 27.9 & 44.1 & 61.9 & \\
\hline
\end{tabular}

${ }^{\text {a }}$ Score: $27-40 \%$ : 1 ; 41-54\%: 3; 55-68\%: 5; 69-82\%: 7, 83-96\%: 9

\section{Discussion}

Salinity stress reduced the root length and root weight of the rice genotypes. But the extent of reduction varied with genotypes and levels of salinity. Root length was reduced by 18 to $93 \%$ with the average of $61.9 \%$ and root dry weight was reduced by 27 to $96 \%$ with an average of $64.3 \%$. Rajakumar (2013) reported that increasing salt stress resulted in gradual decrease in root length. The higher rate of reduction of root length under salinity might be due to elevated inhibitory effect of $\mathrm{NaCl}$ salt to root growth compared with shoot growth (Jamil et al., 2007; Hakim et al., 2010; Anbumalarmathi and Mehta, 2013).

\section{Conclusions}

Salinity stress reduced the root growth of the rice genotypes. The extent of root weight and root length reduction varied with genotypes and levels of salinity.

\section{References}

Anbumalarmathi, J. and Mehta, P. 2013. Effect of salt stress on germination of indica rice varieties. European Journal of Biological Science, 6(1): 1-6.

CCC. (Climate Change Cell). 2007. Climate Change and Bangladesh, Department of Environment, Government of the People's Republic of Bangladesh, Dhaka.

Ferdose J., Kawasaki M., Taniguchi M. and Miyake H. 2009. Differential sensitivity of rice cultivars to salinity and its relation to ion accumulation and root tip structure. Plant Production Science, 12(4): 453-461.

Flowers, T. J. 2004. Improving crop salt tolerance. Journal of Experimental Botany 55: 307-319.

Flowers, T. J. and Yeo, A. R. 1981.Variability in the resistance of sodium chloride salinity within rice (Oryza sativa L.) varieties. New Phytologist, 88: 363-373.

Gholizadeh, F. and Navabpour, S. 2011. Effect of salinity on morphological and physiological characteristics in correlation to selection of salt tolerance in rice (Oryza sativa L.). International Journal of Agricultural Research, 6: 780-788.

Goldani, M. and Latifi, N. 2006. Effect of salinity on germination and seedling growth of three cultivars of wheat. Journal of Agricultural Sciences and Natural Resources, 2: 47-52.
Hakim, M. A.; Juraimi, A. S.; Begum, M.; Hanafi, M. M.; Ismail, M. R. and Selamat, A. 2010. Effect of salt stress and germination and early seedling growth of rice (Oryza sativaL.). African Journal of Biotechnology, 9: 1911-1918.

Hussain, M.; Park, H. W.; Farooq, M.; Jabran, K. and Lee, D. J. 2013. Morphological and physiological basis of salt resistance in different rice genotypes. International Journal of Agriculture and Biology, 15: 113-118.

IRRI. 1996. Standard evaluation system for rice. $4^{\text {th }}$ edn. International Rice Research Institute. Manila, Philippines. p. 52.

Jamil, M.; Lee, K. B.; Jung, K. Y.; Lee, D. B.; Han, M. S. and Rha, E. S. 2007. Salt stress inhibits germination and early seedling growth in cabbage (Brassica oleracea capitata L.). Pakistan Journal of Biological Sciences, 10(6): 910-914.

Kazemi, K. and Eskandari, H. 2013. Effects of salt stress on germination and early seedling growth of rice ( Oryza sativa ) cultivars in Iran. African Journal of Biotechnology, 10(77): 17789-17792.

Kuchanur, P. H.; Patil, S. G.; Dronavallin, P. S.; Pattar and Reddy, B. G. M. 2006. Screening of rice (Oryza sativa) genotypes for salt tolerance in saline Vertislos of Tungabhadra Command, Indian Journal of Agricultural Sciences, 76: 286-288.

Kumar, V.; Shriram, V.; KaviKishor, P. B.; Jawaliand, N. and Shitole, M. G. 2010. Enhanced proline accumulation and salt stress tolerance of transgenic indica rice by over expressing P5CSF129A gene. Plant Biotechnology Reports, 4: 37-48.

Ma, H. K.; Chong, X. W. and Deng. 2007. Rice research past, present and future. Journal of Integrative Plant Biology, 49: 729-730.

Mahmud, S.; Sharmin, S.; Chowdhury, B. L. D. and Hossain, M. A. 2017. Effect of salt stress and alleviating role of methyl jasmonate in some rice varieties. Asian Journal of Plant Sciences, 16(2): 87-93.

Melissa, A.; Susan, F.; McCouch, R. and Hall, R. D. 2009. Not just a grain of rice: the quest for quality. Trends in Plant Science, 14(3): 1360-1385.

Mohammadi-Nejad, G.; Singh, R. K.; Arzani, A.; Rezaie, A. M.; Sabouri, H. and Gregorio, G. B. 2010. Evaluation of salinity tolerance in rice 
genotypes. International Journal of Plant Production, 4(3): 199-208.

Ologundudu, A. F.; Adelusi, A. A. and Akinwale, R. O. 2014. Effect of salt stress on germination and growth parameters of rice (Oryza sativa L.). Notulae Scientia Biologicae, 6(2): 237-243.

Rad, H. E.; Aref, F. and Rezaei, M. 2012. Response of rice to different salinity levels during different growth stages. Research Journal of Applied Sciences, Engineering and Technology, 4(17): 3040-3047.

Rajakumar, R. 2013. A study on effect of salt stress in the seed germination and biochemical parameters of rice (Oryza sativa L.) under in vitro condition. Asian Journal of Plant Science and Research, 3(6): 20-25.

Sheng, X.; Hu, B.; He, Z.; Ma, F.; Feng, J.; Shen, W. and Yang, J. 2011. Enhancement of salinity tolerance during rice seed germination by presoaking with hemoglobin. International Journal of Molecular Sciences, 12: 2488-2501.

Shereen, A.; Mumtaz, S.; Raza, S.; Khan, M. A. and Solangi, S. 2005. Salinity effects on seedling growth and yield components of different inbred rice lines. Pakistan Journal of Botany, 37(1): 131139.

Shrivastava, P. and Kumar, R. 2015. Soil salinity: A serious environmental issue and plant growth promoting bacteria as one of the tools for its alleviation. Saudi Journal of Biological Sciences, 22: 123-131.

Sosa, L.; Llanes, A.; Reinoso, H.; Reginato, M. and Luna, V. 2005. Osmotic and specific ion effects on the germination of Prosopis strombulifera. Annals of Botany, 96: 261-267.

SRDI. 2010. SRMAF Project, Soil Resource Development Institute, Ministry of Agriculture, Government of Bangladesh.

Tavakkoli, E.; Fatehi, F.; Coventry, S.; Rengasamy, P. and McDonald, G. K. 2011. Additive effects of $\mathrm{Na}+$ and $\mathrm{Cl}-$ ions on barley growth under salinity stress. Journal of Experimental Botany, 62: 21892203.

World Bank. 2000. Bangladesh: Climate Change \& Sustainable Development. Report No.21104BD,Dhaka.http://www.wds.worldbank.o rg/external/default/WDSContentServer/WDSP/IB/ 2001/04/13/000094946_01033105302920/Render ed/PDF/multi0page.pdf.

Zeng, L. and Shannon, M. C. 2000. Salinity effects on seedling growth and yield components of rice. Crop Science, 40: 996-1003.

Zhang, H.; Irving, L. J.; McGill, C.; Matthew, C.; Zhou, D. and Kemp, P. 2010. The effects of salinity and osmotic stress on barley germination rate: sodium as an osmotic regulator. Annals of Botany, 106: 1027-1035. 